\title{
Adjuvant Trastuzumab with or without Chemotherapy in Stage 1 pT1N0 HER2+ Breast Cancer: A National Cancer Database Analysis
}

Lifen Cao ( $\sim$ lifencao0929@gmail.com )

University Hospitals Cleveland Medical Center https://orcid.org/0000-0001-9366-7522

Robert Shenk

UH Cleveland Medical Center

Nickolas Stabellini

Case Western Reserve University

Megan E. Miller

UH Cleveland Medical Center

Christopher W. Towe

UH Cleveland Medical Center

Alberto J. Montero

UH Cleveland Medical Center

\section{Research Article}

Keywords: breast cancer, immunotherapy, chemotherapy, HER2 positive, HER2 breast, NCDB

Posted Date: August 24th, 2021

DOI: https://doi.org/10.21203/rs.3.rs-813296/v1

License: () (1) This work is licensed under a Creative Commons Attribution 4.0 International License.

Read Full License

Version of Record: A version of this preprint was published at Breast Cancer Research and Treatment on October 16th, 2021. See the published version at https://doi.org/10.1007/s10549-021-06411-4. 


\section{Abstract}

Purpose: Approximately $20 \%$ of all breast cancers (BC) are HER2 amplified. In the APT trial, weekly paclitaxel/ trastuzumab in node negative HER2+ BC with tumors $<3 \mathrm{~cm}$ was associated with a 7-year invasive disease-free survival of $93 \%$. However, this was in the context of a non-randomized trial, and for pT1NO HER2+ BC it remains unclear whether HER2 monotherapy would provide similar clinical outcomes to chemo-HER2 therapy. We hypothesized that adjuvant chemo-HER2 therapy would be associated with a modestly improved overall survival compared to HER2 monotherapy in patients with tumors $<2 \mathrm{~cm}$.

Methods: In the National Cancer Database (2004-2017), patients with a primary diagnosis of pT1N0M0 HER2+ BC, were separated into two groups: (i) HER2 monotherapy, i.e. trastuzumab, and (ii) chemo-HER2 therapy. A 3:1 propensity match was performed to balance patient selection bias between the two different cohorts. Long-term overall survival (OS) was compared between both groups.

Results: A total of 23, 281 patients met the criteria. 22,268 (96.7\%) received chemo-HER2 therapy and $1,013(4.4 \%)$ received HER2 monotherapy. Propensity match identified 1,995 patients who received chemo-HER2 therapy, and 666 who received HER2 monotherapy. After match, adjuvant chemo-HER2 therapy was associated with a modest survival advantage over HER2 monotherapy (5-year OS 94.1\% vs. $90.6 \%, P=0.041)$.

Conclusions: Even though there is a modest OS advantage favoring adjuvant chemo-HER2 therapy in pT1NO HER2+ BC, HER2 monotherapy was associated with 5-year OS $>90 \%$. Therefore, in select patients who have contraindications for cytotoxic chemotherapy, or decline adjuvant chemotherapy, adjuvant trastuzumab monotherapy appears to be a reasonable alternative.

\section{Introduction}

Breast cancer (BC) is the most commonly occurring cancer and the leading cause of cancer death among women worldwide. In the U.S. alone, an estimated 330,840 new cases of BC will be diagnosed in 2021 [1]. HER2-postive BC represents $20-25 \%$ of BC worldwide [2], and have a distinctive biology of amplification of the HER2 oncogene, which prior to the development of HER-2 targeting agents was associated with a higher risk of recurrence and poorer clinical outcomes [3-6].

The development of trastuzumab, a humanized monoclonal HER-2 specific antibody was considered a landmark achievement in the field of targeted therapy in the 1990s [7]. When combined with chemotherapy, trastuzumab improves progression-free survival and overall survival in metastatic HER2positve BC [8], and therapies inhibiting HER2 signaling together with chemotherapy have become the standard of care [9].

Trastuzumab based-therapy is well established for early stage HER2 + breast cancer, however in patients with small stage 1 tumors, the data is limited since they were excluded from the initial pivotal adjuvant trastuzumab trials [10]. The APT trial examined a less intensive chemotherapy regimen in a non- 
randomized phase 2 trial [11]. This study enrolled patients with HER 2 + node negative cancers $<3 \mathrm{~cm}$ who all received weekly paclitaxel with trastuzumab, and has reported 3-year and 7-year invasive disease-free survival (iDFS) of $98.7 \%$ and $93 \%$, respectively $[10,11]$. There are no randomized adjuvant trials evaluating trastuzumab alone without chemotherapy in patients with HER2-positive node negative $2 \mathrm{~cm}$ or smaller.

There are also limited data comparing the impact of modern chemo-HER2 adjuvant therapies compared to trastuzumab mono-therapy which is still a relevant question for lower risk node negative stage 1 HER2 + patients. Based on the Aphinity trial the addition of pertuzumab to standard adjuvant therapy in HER2 + early $B C$ should be limited only to patients with node-positive disease $[12,13]$.

A recently published non-inferiority trial investigated the relative value of adjuvant trastuzumab monotherapy in older patients with stages I-III HER2 + early breast cancer [14]. Although the primary objective of non-inferiority for trastuzumab monotherapy was not met, less than a percentage point difference for overall survival at 3 years was observed between trastuzumab-chemotherapy and trastuzumab monotherapy groups $(97.2 \%$ vs. $96.6 \%$; (HR, $1.07 ; 95 \% \mathrm{Cl}, 0.36$ to 3.19$))$. However, this trial was not designed to address the question of the relative value of chemotherapy added to trastuzumab mono-therapy in pT1NO HER2 + BC at the lower end of the risk spectrum regardless of age.

Using the National Cancer Database (NCDB), the largest source of BC data in the United States, our research aim was to determine whether adjuvant HER2 antibody therapy, i.e. trastuzumab was associated with similar overall survival rates compared to chemo plus trastuzumab in patients with pT1N0M0 HER2 + BC. Prior to 2003, the NCDB categorized trastuzumab as chemotherapy. From 2003 onwards, trastuzumab and pertuzumab were categorized as immunotherapy. Pertuzumab was not FDA approved as adjuvant therapy for HER2 + breast cancer until 2017 [15].Therefore, we confined our query to 20032017 when adjuvant immunotherapy for pT1N0 HER2 + breast cancer could only be trastuzumab. We hypothesized that the combination of chemotherapy and trastuzumab (chemo-HER2 therapy) would result in only a very modest survival advantage over trastuzumab monotherapy, which if true, would provide real world evidence supporting use of trastuzumab as adjuvant monotherapy in patients considered poor candidates for adjuvant chemotherapy.

\section{Methods}

Data collection and Data elements:

A retrospective cohort study of the National Cancer Database (NCDB) was performed. Jointly sponsored by the American College of Surgeons and the American Society, NCDB is a clinical oncology database sourced from hospital registry data representing more than $70 \%$ of newly diagnosed cancer cases nationwide. The database covers more than 1,500 Commission on Cancer (CoC)-accredited facilities. Definition of the database variables are available from the dictionary of NCDB Participant Use Data File (http://ncdbpuf.facs.org). The CoC's NCDB and the hospitals participating in the CoC NCDB are the 
source of the de-identified data used herein; they have not verified and are not responsible for the statistical validity of the data analysis or the conclusions derived by the authors.

Patient Cohort and Data analysis:

The NCDB was queried to analyze patients with HER2 + pT1NOMO BC who received surgery from 2013 to 2017. Pathological staging data for the cohort was based on TNM classification in American Joint Committee on Cancer (AJCC) 7th edition [16]. Patients were excluded if they had metastasis, hormone receptor positive but did not receive endocrine therapy or were missing critical study information (e.g., pathologic stage or HER2 status).

The cohort was categorized by treatment status as immunotherapy alone (HER2 monotherapy) or immunotherapy plus chemotherapy (chemo-HER2 therapy). In the context of stage 1 HER2 + breast cancer, the only immunotherapy agent that patients could have received is trastuzumab, since pertuzumab was not FDA approved for use as adjuvant therapy for HER2 + breast cancer until 2017. The primary outcome was overall survival .

Analysis included univariate comparison of patient factors associated with chemo-HER2 therapy vs. HER2 monotherapy). To compare the two groups, Wilcoxon rank-sum test was utilized for continuous variables and chi-square for categorical data. Multivariable logistic regression determined the association between adjuvant chemotherapy and demographic/tumor factors. Overall survival (OS) differences between the two groups was analyzed using Kaplan-Meier survival estimates and compared by utilizing a log-rank test. To control for confounding effects, cox proportional hazard analysis was also performed. To further account for differences between patient cohorts receiving chemo-HER2 therapy vs. HER2 monotherapy, a 3:1 propensity match was performed, correcting for age, race, Charlson score, insurance, facility, tumor grade, hormone receptor status, mastectomy procedures, lymph nodes removed, lymphovascular invasion status, and sub pathological T1 stage (T1a, T1b, T1c). The propensity match dropped observations of patients receiving HER2 monotherapy whose propensity score as higher than the maximum or less than the minimum score of the controls (chemo-HER2 therapy). 3:1 nearest neighbor matching replacement was used, with the caliper set to 0.01 . Standardized differences before and after matching were reported and graphed during the analysis of propensity matching. Differences in survival among the matched pairs were analyzed using a stratified log-rank test and Cox proportional hazard regression analysis using a clustered "sandwich" robust variance estimator to account for clustering within the matched pairs.

All statistical analysis was performed using STATA/MP, version 16.0 (Stata Corp LLC, College Station, TX). Institutional Review Board (IRB) approval was exempted by the University Hospitals Cleveland Medical Center IRB as all data is de-identified.

\section{Results}


Clinical and demographic details. The final analytic cohort included 23, 281 patients with a pathological diagnosis of pT1NOM0 stage BC (Table 1). 22,268 (96.7\%) received adjuvant chemo-HER2 therapy and $1,013(4.4 \%)$ received HER2 monotherapy. As expected, patients who received adjuvant chemo-HER2 therapy were more likely to have higher grade tumors (poor or non-differentiated ( $53.2 \%$ vs. $45.7 \%, p<$ $0.001)$, with lympho-vascular invasion $(13.9 \%$ vs. $9.7 \%, p<0.001)$ compared to patients who received HER2 monotherapy. Patients who received chemo-HER2 therapy were more frequently non-white $(18.2 \%$ vs. $15.0 \%, p=0.027)$, had private insurance $(65.6 \%$ vs. $43.0 \%, p<0.001)$, and were treated at academic/integrated facilities ( $49.2 \%$ vs. $45.5 \%, P=0.001)$, There were no differences in comorbidity, distance to the hospital, or community designation. By contrast, patients who received HER2 monotherapy were more likely to have smaller tumors $(p=0.019)$, that were more often $H R+$ and received adjuvant endocrine therapy $(p<0.001)$. 
Table 1

Demographic and clinical characteristics of HER2 + pT1N0M0 breast cancer patients, NCDB 2013-2017

\section{CHARACTERISTICS}

HER2 monotherapy

$(n=1,013)$
NO. $\%$

$66(35-90)$
Chemo-HER2 therapy

$(n=22,268)$

\begin{tabular}{|c|c|c|c|c|c|}
\hline & NO. & $\%$ & NO. & $\%$ & $\begin{array}{l}P \\
\text { value }\end{array}$ \\
\hline Age, years & \multicolumn{2}{|c|}{$66(35-90)$} & \multicolumn{2}{|c|}{$57(29-90)$} & $\begin{array}{l}\mathrm{P}< \\
0.001\end{array}$ \\
\hline Race & & & & & $\begin{array}{l}P= \\
0.027\end{array}$ \\
\hline White & 859 & 85.05 & 18,076 & 81.81 & \\
\hline Black & 92 & 9.11 & 2,557 & 11.57 & \\
\hline Asian and other & 59 & 5.84 & 1,463 & 6.62 & \\
\hline Charlson-Deyo Score & & & & & $\begin{array}{l}P= \\
0.283\end{array}$ \\
\hline 0 & 851 & 84.01 & 19,141 & 85.96 & \\
\hline 1 & 128 & 12.64 & 2,543 & 11.42 & \\
\hline 2 & 26 & 2.57 & 430 & 1.93 & \\
\hline 3 & 8 & 0.79 & 154 & 0.69 & \\
\hline Insurance & & & & & $\begin{array}{l}P< \\
0.001\end{array}$ \\
\hline Public & 564 & 56.23 & 7,251 & 32.83 & \\
\hline Private & 431 & 42.97 & 14,483 & 65.58 & \\
\hline Not insured & 8 & 0.8 & 352 & 1.59 & \\
\hline Facility Type & & & & & $\begin{array}{l}P= \\
0.001\end{array}$ \\
\hline Community cancer program & 110 & 11.1 & 1,658 & 8.13 & \\
\hline $\begin{array}{l}\text { Comprehensive community cancer } \\
\text { program }\end{array}$ & 430 & 43.39 & 8,701 & 42.69 & \\
\hline Academic/research program & 333 & 33.6 & 6,933 & 34.02 & \\
\hline Integrated network cancer program & 118 & 11.91 & 3,090 & 15.16 & \\
\hline
\end{tabular}

*only patients who received endocrine therapy were included 


\begin{tabular}{|c|c|c|c|c|c|}
\hline CHARACTERISTICS & & $\begin{array}{l}\text { HER2 } \\
\text { monotherapy } \\
(n=1,013)\end{array}$ & & $\begin{array}{l}\text { Chemo-HER2 } \\
\text { therapy } \\
(n=22,268)\end{array}$ & \\
\hline Facility Area & & & & & $\begin{array}{l}P= \\
0.755\end{array}$ \\
\hline Metro & 848 & 86.71 & 18,897 & 86.94 & \\
\hline Urban & 113 & 11.55 & 2,524 & 11.61 & \\
\hline Rural & 17 & 1.74 & 314 & 1.44 & \\
\hline Distance to the hospital (miles) & & & & & $\begin{array}{l}P= \\
0.089\end{array}$ \\
\hline $0.1 / 20$ & 651 & 74.23 & 14,146 & 74.48 & \\
\hline $20.1 / 40$ & 111 & 12.66 & 2,833 & 14.92 & \\
\hline $40.1 / 60$ & 52 & 5.93 & 895 & 4.71 & \\
\hline $60.1 / \max$ & 63 & 7.18 & 1,115 & 5.87 & \\
\hline Grade & & & & & $\begin{array}{l}P< \\
0.001\end{array}$ \\
\hline Well differentiated & 79 & 8.09 & 1,115 & 5.21 & \\
\hline Moderately differentiated & 451 & 46.21 & 8,905 & 41.61 & \\
\hline Poorly differentiated & 445 & 45.59 & 11,353 & 53.04 & \\
\hline Undifferentiated & 1 & 0.1 & 30 & 0.14 & \\
\hline Lympho-vascular invasion & & & & & $\begin{array}{l}P< \\
0.001\end{array}$ \\
\hline Not Present & 807 & 90.27 & 16,271 & 86.08 & \\
\hline present & 87 & 9.73 & 2,631 & 13.92 & \\
\hline Hormone Receptor & & & & & $\begin{array}{l}P< \\
0.001\end{array}$ \\
\hline Not Present & 230 & 22.7 & 6,172 & 27.72 & \\
\hline Present* & 783 & 77.3 & 16,096 & 72.28 & \\
\hline Sub T stage & & & & & $\begin{array}{l}P= \\
0.019\end{array}$ \\
\hline $1 a$ & 170 & 17.38 & 3,222 & 15.21 & \\
\hline
\end{tabular}

*only patients who received endocrine therapy were included 


\begin{tabular}{|lllll|}
\hline CHARACTERISTICS & & $\begin{array}{l}\text { HER2 } \\
\text { monotherapy } \\
(\mathbf{n = 1 , 0 1 3 )}\end{array}$ & $\begin{array}{l}\text { Chemo-HER2 } \\
\text { therapy } \\
(\mathbf{n}=\mathbf{2 2 , 2 6 8 )}\end{array}$ \\
\hline 1b & 285 & 29.14 & 5,688 & 26.85 \\
\hline 1c & 523 & 53.48 & 12,272 & 57.94 \\
\hline *only patients who received endocrine therapy were included & & \\
\hline
\end{tabular}

Significant differences in the types of breast surgery were observed between the two different adjuvant therapy groups. Patients who received HER2 monotherapy, were significantly more likely to undergo a partial mastectomy $(p<0.001)$, and had fewer axillary lymph nodes removed $(p<0.002$, Table 2$)$ compared to patients who received adjuvant chemo-HER2 therapy.

Table 2

Surgical and adjuvant treatment for HER2 + pT1N0M0 breast cancer patients (NCDB 2013-2017).

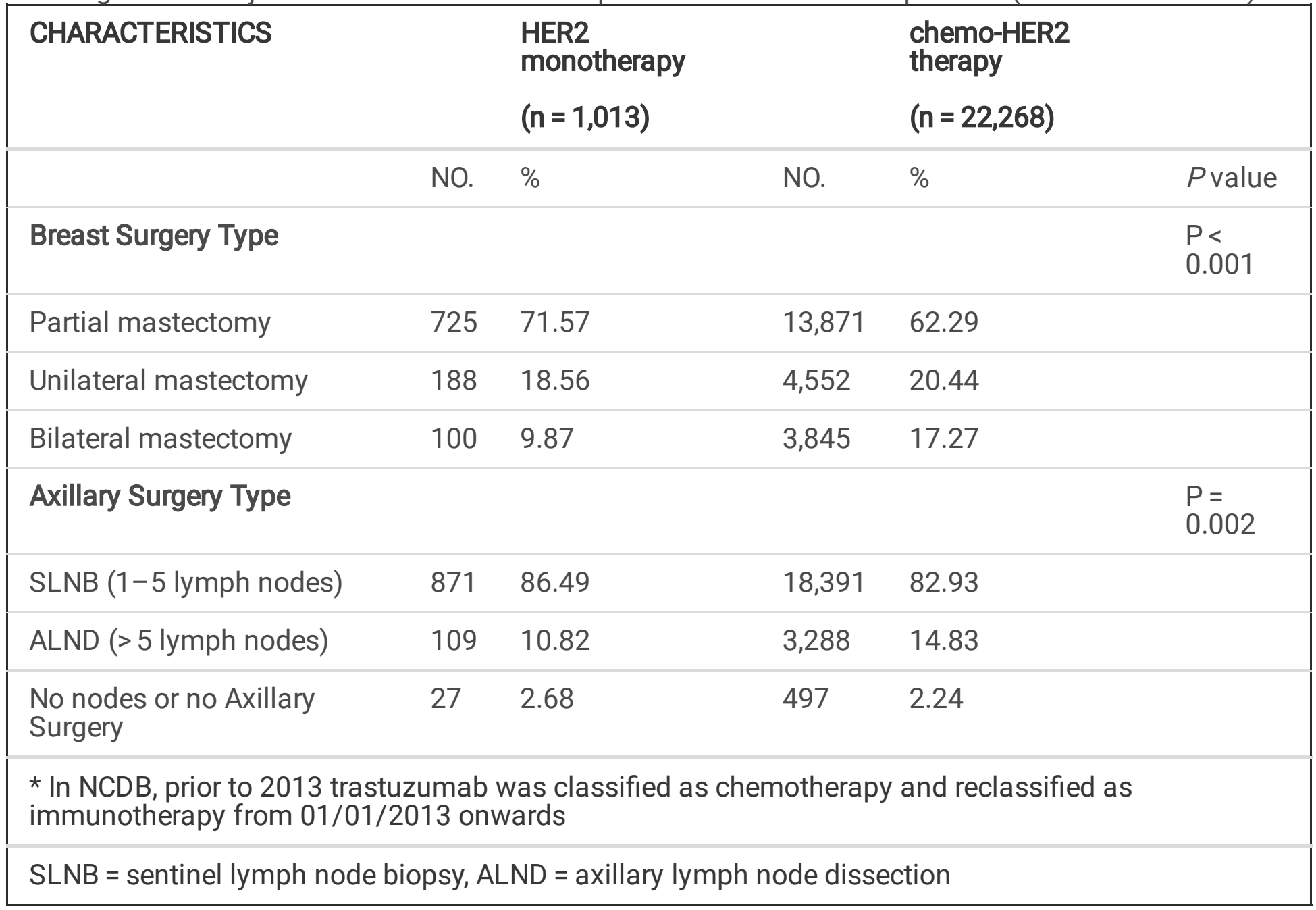

Factors associated with chemo-HER2 therapy. A multivariable logistic regression was performed to determine clinical factors independently associated with receipt of chemo-HER2 therapy (Table 3). Patients with $\mathrm{T} 1 \mathrm{~b}$ and $\mathrm{T} 1 \mathrm{c}$ BC were more likely to receive chemotherapy as compared to those with $\mathrm{T} 1 \mathrm{a}$ 
disease $(O R=1.468$ and $O R=1.885, p \leq 0.001)$. Other factors significantly associated with an increased likelihood of receiving adjuvant chemo-HER2 therapy included treatment at an academic/integrated facility or comprehensive community cancer program, poorly differentiated or undifferentiated histology. Older age $(O R=0.924, p<0.001)$ and hormone receptor positive with endocrine therapy $(O R=0.659, p<$ $0.001)$ were less likely to receive chemo-HER2 therapy. 
Table 3

Multivariable logistic regressions for predictors of receipt of adjuvant chemo-HER2 vs. HER2 monotherapy in HER2 + pT1 NOMObreast cancer patients (NCDB 20132017).

\begin{tabular}{|c|c|c|c|c|}
\hline \multirow{2}{*}{$\begin{array}{l}\text { chemo-HER2 vs. HER2 monotherapy } \\
\text { Age }\end{array}$} & \multirow{2}{*}{$\begin{array}{l}\text { Odds Ratio } \\
0.924\end{array}$} & \multicolumn{2}{|c|}{$95 \%$ Conf. Int. } & \multirow{2}{*}{$\begin{array}{l}\text { p-value } \\
<0.001\end{array}$} \\
\hline & & 0.915 & 0.933 & \\
\hline \multicolumn{5}{|l|}{ Race } \\
\hline White & Reference & & & \\
\hline African American & 1.040 & 0.808 & 1.339 & 0.760 \\
\hline Asian or others & 0.864 & 0.628 & 1.190 & 0.371 \\
\hline \multicolumn{5}{|l|}{ Charlson-Deyo score } \\
\hline 0 & Reference & & & \\
\hline 1 & 1.076 & 0.869 & 1.332 & 0.502 \\
\hline 2 & 1.116 & 0.706 & 1.763 & 0.638 \\
\hline 3 & 1.641 & 0.711 & 3.785 & 0.245 \\
\hline \multicolumn{5}{|l|}{ Facility Type } \\
\hline Community & Reference & & & \\
\hline Comprehensive & 1.339 & 1.051 & 1.705 & 0.018 \\
\hline Academic/Integrated & 1.329 & 1.044 & 1.692 & 0.021 \\
\hline \multicolumn{5}{|l|}{ Insurance Status } \\
\hline \multicolumn{5}{|l|}{ Public insurance } \\
\hline Private insurance & 1.023 & 0.850 & 1.230 & 0.811 \\
\hline Not insured & 1.186 & 0.516 & 2.724 & 0.688 \\
\hline \multicolumn{5}{|l|}{ Grade } \\
\hline Well differentiated & Reference & & & \\
\hline Moderately differentiated & 1.159 & 0.872 & 1.541 & 0.309 \\
\hline Poorly or undifferentiated & 1.398 & 1.046 & 1.869 & 0.024 \\
\hline HR & 0.659 & 0.550 & 0.791 & $<0.001$ \\
\hline Lympho-vascular invasion & 1.264 & 0.991 & 1.613 & 0.059 \\
\hline \multicolumn{5}{|l|}{ Sub T stage } \\
\hline T1a & & & & \\
\hline
\end{tabular}




\begin{tabular}{|lllll|}
\hline chemo-HER2 vs. HER2 monotherapy & Odds Ratio & \multicolumn{2}{l|}{ 95\% Conf. Int. } & p-value \\
\hline T1b & 1.468 & 1.173 & 1.835 & 0.001 \\
\hline T1c & 1.885 & 1.533 & 2.318 & $<0.001$ \\
\hline
\end{tabular}

\section{Survival analyses}

Initial Kaplan-Meier estimates demonstrated that among patients who underwent definitive breast surgery those who received adjuvant HER2 monotherapy had a significantly lower OS relative to patients who received adjuvant chemo-HER2 therapy $(p<0.001)$. Both 3-year OS $(98.7 \%$ vs. $97.0 \%)$ and 5 -year OS $(96.7 \%$ vs. $90.6 \%)$ were higher in patients who received chemo-HER2 therapy Kaplan Meier estimate of OS is shown in Fig. 1.

Propensity matching was performed, which generated 1,995 chemo-HER2 and 666 HER2 monotherapy patients. Propensity matching effectively reduced selection bias between both groups (Fig. 2 and Supplemental Table 1). In the matched cohort, HER2 monotherapy was associated with poorer overall survival $(P=0.041$, Fig. 3 ) compared to the adjuvant chemo-HER2 therapy group. The median follow up time was 39.4 months vs. 38.9 months for HER2 monotherapy and chemo-HER2 therapy, respectively.

On multivariable Cox proportional hazard regression, the survival benefit of chemo-HER2 therapy was no longer statistically significant but trending (HR 0.655, $p=0.068$, Table 4) when controlling for demographic, clinical pathologic, and tumor factors. African American $(H R=1.995, p=0.024)$ and high comorbidity $(H R=4.320, p<0.001)$ were significant predictors of increased mortality. 
Table 4

Cox proportional hazard regression for overall survival of HER2 + pT1NOMO patients among propensity matched cohort.

\begin{tabular}{|c|c|c|c|c|}
\hline \multirow[b]{2}{*}{ chemo-HER2 vs. HER2 monotherapy } & \multirow{2}{*}{$\begin{array}{l}\text { Hazard Ratio } \\
0.655\end{array}$} & \multicolumn{2}{|c|}{ 95\% Conf. Int. } & \multirow{2}{*}{$\begin{array}{l}\text { p-value } \\
0.068\end{array}$} \\
\hline & & 0.416 & 1.033 & \\
\hline Age & 1.031 & 0.999 & 1.064 & 0.060 \\
\hline \multicolumn{5}{|l|}{ Race } \\
\hline White & Reference & & & \\
\hline African American & 1.995 & 1.096 & 3.635 & 0.024 \\
\hline Asian or others & 1.179 & 0.440 & 3.159 & 0.743 \\
\hline \multicolumn{5}{|l|}{ Charlson-Deyo score } \\
\hline 0 & Reference & & & \\
\hline 1 & 1.648 & 0.929 & 2.925 & 0.088 \\
\hline 2 & 4.320 & 1.908 & 9.782 & $<0.001$ \\
\hline 3 & 2.464 & 0.274 & 22.178 & 0.421 \\
\hline \multicolumn{5}{|l|}{ Facility Type } \\
\hline Community & Reference & & & \\
\hline Comprehensive & 0.722 & 0.374 & 1.394 & 0.332 \\
\hline Academic & 0.614 & 0.318 & 1.185 & 0.146 \\
\hline Integrated & 0.932 & 0.420 & 2.067 & 0.862 \\
\hline \multicolumn{5}{|l|}{ Insurance Status } \\
\hline Public insurance & Reference & & & \\
\hline Private insurance & 0.464 & 0.227 & 0.949 & 0.036 \\
\hline \multicolumn{5}{|l|}{ Grade } \\
\hline Well differentiated & Reference & & & \\
\hline Moderately differentiated & 1.216 & 0.472 & 3.134 & 0.685 \\
\hline Poorly or undifferentiated & 1.039 & 0.395 & 2.736 & 0.938 \\
\hline HR & 0.535 & 0.308 & 0.930 & 0.027 \\
\hline Lympho-vascular invasion & 1.089 & 0.498 & 2.381 & 0.830 \\
\hline
\end{tabular}




\begin{tabular}{|lllll|}
\hline & Hazard Ratio & 95\% Conf. Int. & p-value \\
\hline T1a & Reference & & & \\
\hline T1b & 0.910 & 0.459 & 1.802 & 0.786 \\
\hline T1c & 0.849 & 0.451 & 1.597 & 0.611 \\
\hline
\end{tabular}

\section{Discussion}

For node negative early HER2 + BC, adjuvant weekly paclitaxel and trastuzumab has emerged as the preferred regimen [11]. The seven-year follow-up analysis of the APT trial reported a 7-year DFS and OS of $93 \%$ (95\% Cl, 90.4 to 96.2$)$ and $95 \%(95 \% \mathrm{Cl}, 92.4$ to 97.7$)$, respectively [10]. More recently, the ATEMPT trial [17] in stage 1 HER2 + BC demonstrated that adjuvant therapy with the anti-body drug conjugate transtuzumab emtansine (T-DM1) was associated with similar survival outcomes as paclitaxel plus trastuzumab $(\mathrm{TH})$ there are important differences in treatment related adverse events. However, since TDM1, is antibody delivered chemotherapy, the question remains what outcomes with trastuzumabmonotherapy are compared to chemo-HER2 therapy-whether TP or T-DM1 in pT1N0 HER2 + early BC.

Our data utilizes the NCDB to address this gap in our clinical knowledge with real world data. There are obvious reasons why chemo-HER2 therapy would be selected in this dataset over trastuzumab monotherapy-younger age, ER- status, higher grade, greater T1 sub-stage. To address these confounding factors we performed propensity matching, and although the overall benefit of chemo-HER2 therapy over trastuzumab monotherapy decreased after matching, the difference still remained clinically and statistically significant.

Our study has several limitations relating to factors not captured in large retrospective cohorts such as the NCDB. The database does not include local regional recurrence or disease-free survival; therefore, our analysis of long-term outcomes is limited only to OS. Similarly, granular details about chemotherapy or immunotherapy agents, doses, combinations, toxicities and decision-making is not included and therefore cannot be factored into analyses. Since pertuzumab was not FDA approved for adjuvant use until 2017, and our analysis was limited to 2003-2017, it is reasonable to deduce that the only other HER2 antibody therapy that patients could have received during this time, outside of a clinical trial was trastuzumab.

Despite these limitations, this analysis provides real world data demonstrating that even in PT1NO HER2 $+\mathrm{BC}$, there does seem to be a modest OS advantage to the addition of adjuvant chemotherapy to trastuzumab. Based on the very good outcomes observed with TH, the standard of care for pT1N0 HER2 $+\mathrm{BC}$ should remain adjuvant chemo-HER2 therapy with TH. The ATEMPT trial suggests that T-DM1 is an alternative. However, in patients who are considered unfit for either TH or T-DM1, or who decline chemotherapy, these data provide evidence that trastuzumab alone is a viable alternative with 5-year OS rate of $90.6 \%$. 


\section{Declarations}

CW Towe reports that he is a consultant for Zimmer Biomet, Astra Zeneca, Atricure, and Medtronic, but that these relationships have not affected this manuscript or the accuracy of the data analysis. CW Towe reports that he has received unrelated research funding from Zimmer Biomet. All other authors have no disclaimers, sources of funding, or financial relationships to declare.

\section{References}

1. Society AC (2021) Cancer Facts and Figs. 2021. https://www.cancer.org/research/cancer-factsstatistics/all-cancer-facts-figures/cancer-facts-figures-2021.html:Accessed 9 August 2021

2. Arteaga CL, Sliwkowski MX, Osborne CK et al (2011) Treatment of HER2-positive breast cancer: current status and future perspectives. Nat Rev Clin Oncol 9(1):16-32. https://doi.org/10.1038/nrclinonc.2011.177

3. Mahtani R, Holmes FA, Badve S et al (2020) A Roundtable Discussion of the Breast Cancer Therapy Expert Group (BCTEG): Clinical Developments and Practice Guidance on Human Epidermal Growth Factor Receptor 2 (HER2)-positive Breast Cancer. Clin Breast Cancer 20(3):e251-e260. https://doi.org/10.1016/j.clbc.2019.08.001

4. Slamon DJ, Clark GM, Wong SG et al (1987) Human breast cancer: correlation of relapse and survival with amplification of the HER-2/neu oncogene. Science 235(4785):177-182. https://doi.org/10.1126/science.3798106

5. Slamon DJ, Godolphin W, Jones LA et al (1989) Studies of the HER-2/neu proto-oncogene in human breast and ovarian cancer. Science 244(4905):707-712. https://doi.org/10.1126/science.2470152

6. Sledge GW Jr (2018) HER2-positive breast cancer. The Breast.763-768

7. Harries M, Smith I (2002) The development and clinical use of trastuzumab (Herceptin). Endocr Relat Cancer 9(2):75-85. https://doi.org/10.1677/erc.0.0090075

8. Makhou I (2018) Therapeutic strategies for breast cancer. The Breast.315-330

9. Mitri Z, Constantine T, O'Regan R (2012) The HER2 Receptor in Breast Cancer: Pathophysiology, Clinical Use, and New Advances in Therapy. Chemother Res Pract 2012:743193.https://doi.org/10.1155/2012/743193

10. Tolaney SM, Guo H, Pernas S et al (2019) Seven-Year Follow-Up Analysis of Adjuvant Paclitaxel and Trastuzumab Trial for Node-Negative, Human Epidermal Growth Factor Receptor 2-Positive Breast Cancer. J Clin Oncol 37(22):1868-1875. https://doi.org/10.1200/JC0.19.00066

11. Tolaney SM, Barry WT, Dang CT et al (2015) Adjuvant paclitaxel and trastuzumab for node-negative, HER2-positive breast cancer. N Engl J Med 372(2):134-141.

https://doi.org/10.1056/NEJMoa1406281

12. Piccart M, Procter M, Fumagalli D et al (2021) Adjuvant Pertuzumab and Trastuzumab in Early HER2Positive Breast Cancer in the APHINITY Trial: 6 Years' Follow-Up. J Clin Oncol 39(13):1448-1457. https://doi.org/10.1200/JC0.20.01204 
13. von Minckwitz G, Procter M, de Azambuja E et al (2017) Adjuvant Pertuzumab and Trastuzumab in Early HER2-Positive Breast Cancer. N Engl J Med 377(2):122-131. https://doi.org/10.1056/NEJMoa1703643

14. Sawaki M, Tokudome N, Mizuno T et al (2011) Evaluation of trastuzumab without chemotherapy as a post-operative adjuvant therapy in HER2-positive elderly breast cancer patients: randomized controlled trial [RESPECT (N-SAS BC07)]. Jpn J Clin Oncol 41(5):709-712. https://doi.org/10.1093/jjco/hyr011

15. Howie LJ, Scher NS, Amiri-Kordestani L et al (2019) FDA Approval Summary: Pertuzumab for Adjuvant Treatment of HER2-Positive Early Breast Cancer. Clin Cancer Res 25(10):2949-2955. https://doi.org/10.1158/1078-0432.CCR-18-3003

16. Edge SB, Compton CC (2010) The American Joint Committee on Cancer: the 7th edition of the AJCC cancer staging manual and the future of TNM. Ann Surg Oncol 17(6):1471-1474. https://doi.org/10.1245/s10434-010-0985-4

17. Tolaney SM, Tayob N, Dang C et al (2021) Adjuvant Trastuzumab Emtansine Versus Paclitaxel in Combination With Trastuzumab for Stage I HER2-Positive Breast Cancer (ATEMPT): A Randomized Clinical Trial. J Clin Oncol 39(21):2375-2385. https://doi.org/10.1200/JC0.20.03398

\section{Figures}




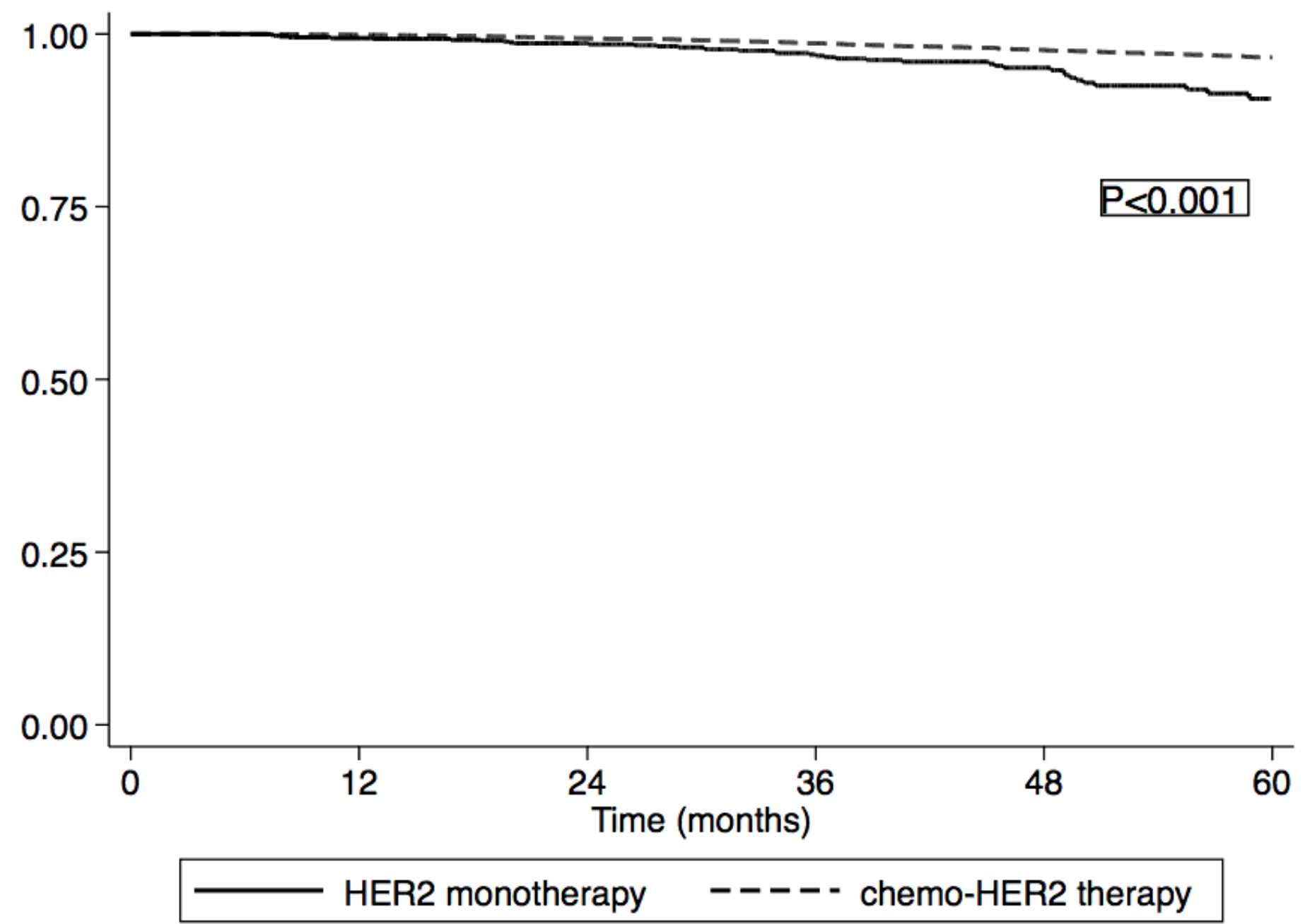

Figure 1

Unadjusted Kaplan-Meier analysis of overall survival of HER2+ pT1NOM0 breast cancer patients comparing chemo-HER2 therapy vs. vs. HER2 monotherapy. Both 3-year and 5-year OS were superior in patients who received chemo-HER2 therapy vs. HER2 monotherapy $(P<0.001): 98.7 \%$ vs. $97.0 \%$, and $96.6 \%$ vs. $90.6 \%$, respectively. 


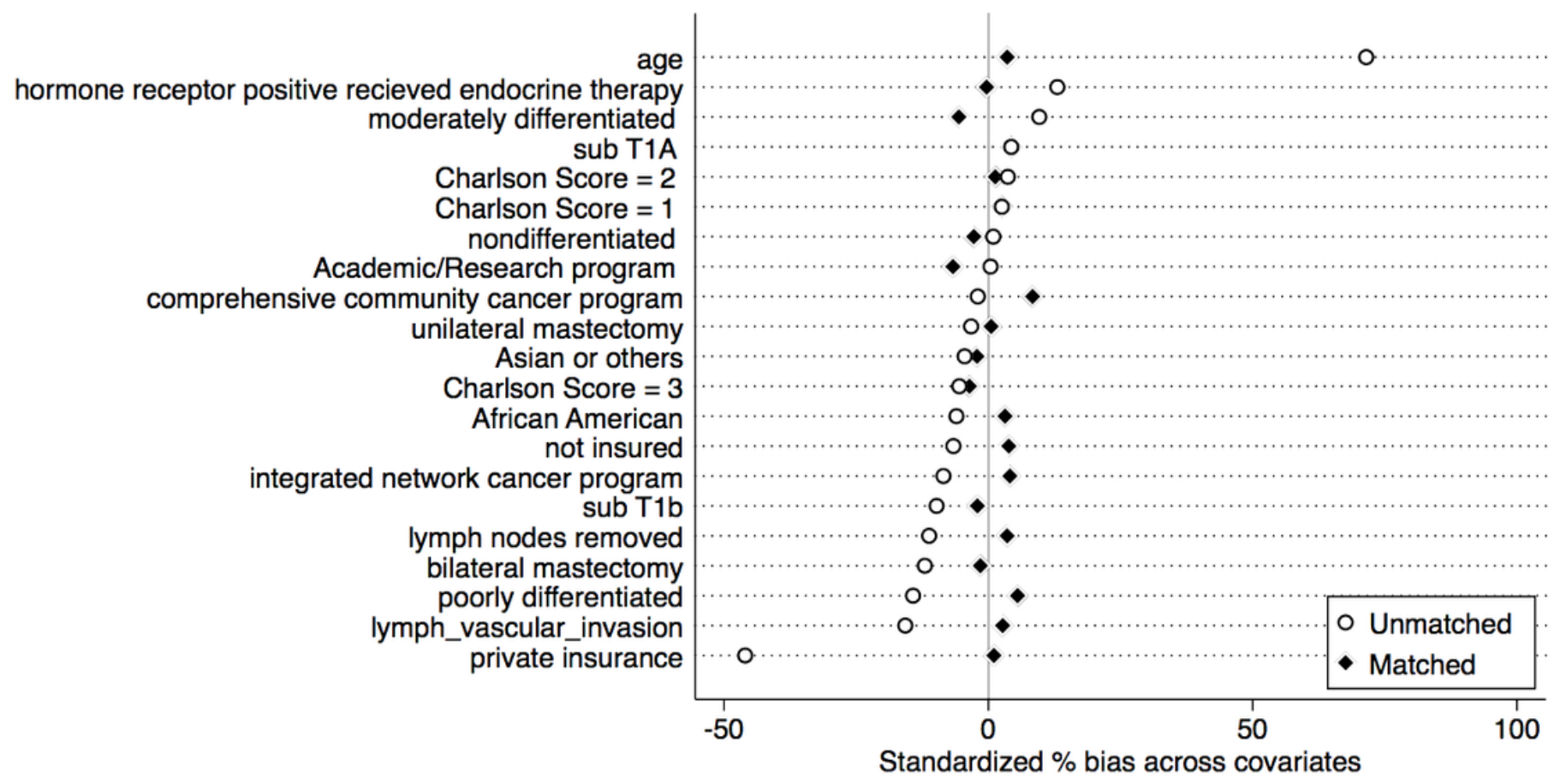

Figure 2

Propensity Matching. Percent bias of unmatched (circle) compared to matched groups (diamond) is shown below. Bias between HER2-chemotherapy and HER2 monotherapy groups was reduced by propensity matching. 


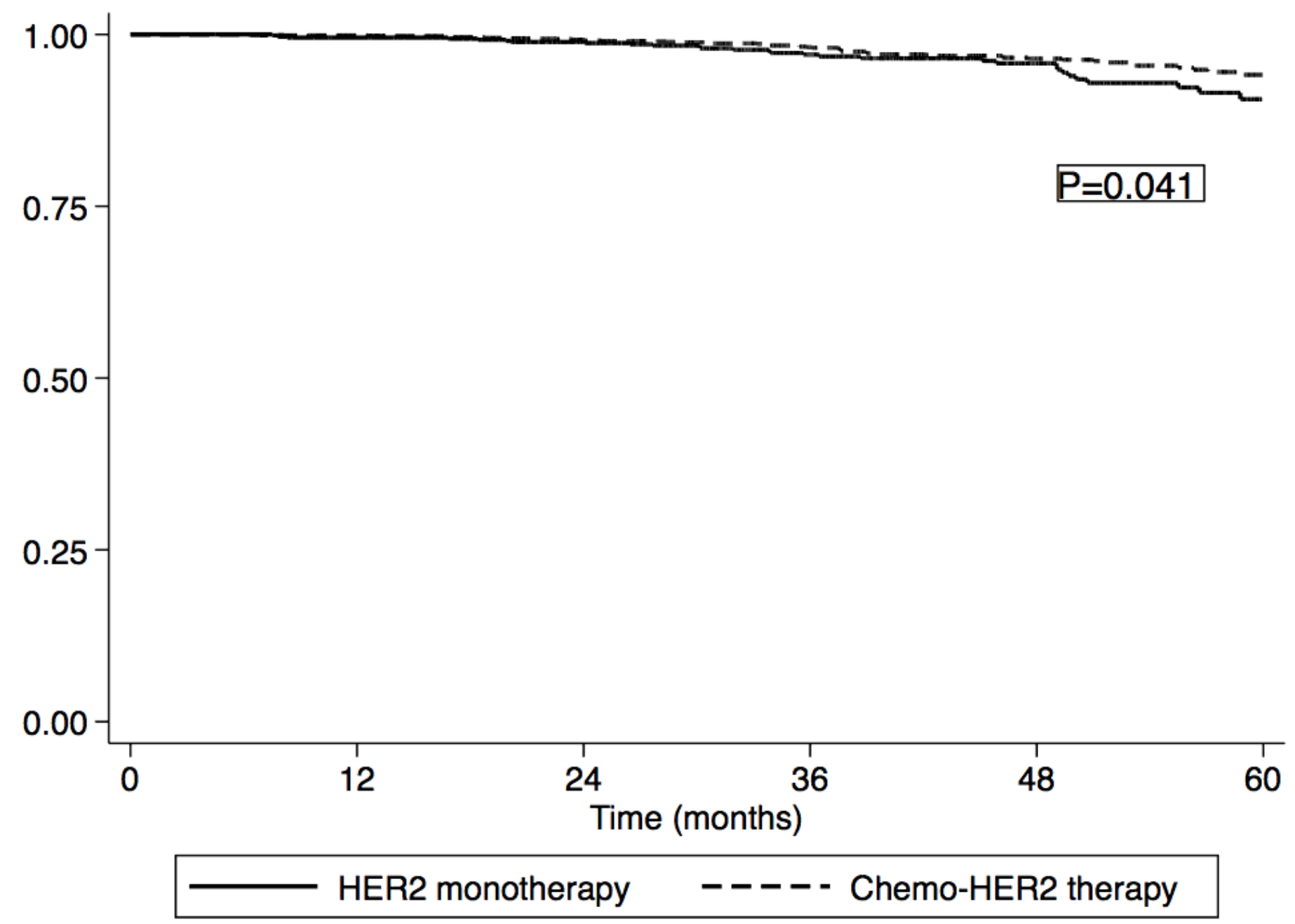

Figure 3

Kaplan-Meier estimates of OS for the propensity-matched group of HER2+ pT1NOMO BC patients. OS at 3 and 5 years were $98.1 \%$ and $94.1 \%$ for the chemo-HER2 therapy cohort compared to $97.1 \%$ and $90.6 \%$, respectively for the HER2 monotherapy group $(P=0.041)$. 